\title{
Improving image compression through the use of evolutionary computing algorithms
}

\author{
O. López-Granado ${ }^{1}$, V. Galiano ${ }^{1}$, A. Martî2 ${ }^{2}$ H. Migallón ${ }^{1}$, \\ M. Martínez-Rach ${ }^{1}$, P. Piñol ${ }^{1} \&$ M.P. Malumbres ${ }^{1}$ \\ ${ }^{1}$ Miguel Hernandez University, Spain \\ ${ }^{2}$ Universidad Politécnica de Valencia, Spain
}

\begin{abstract}
Discrete Wavelet Transform has proved to be powerful for image compression because it is able to compact frequency and spatial localization of image energy into a small fraction of coefficients. For a long time it was assumed that there is no compression gain when coding the sign of wavelet coefficients. However, several attempts were carried out and several image encoders like JPEG 2000 include sign coding capabilities. In this paper, we analyze the convenience of including sign coding techniques in tree-based wavelet image encoders, showing their benefits (bit-rate saving). In order to exploit the scarce redundancy of wavelet coefficients sign, we propose the use of machine learning approaches, like evolutionary algorithms, to find the best sign prediction scheme that maximizes the resulting compression rate. We have developed a sign prediction module based on the results provided by the evolutionary algorithms, which it is able to work with whatever the tree-based wavelet encoder like SPIHT, LTW, and others. After performing several experiments, we have observed that, by including the proposed sign coding capabilities, the sign compression gain is up to $17 \%$. These results show that sign coding techniques are of interest for improving compression rate, especially when working with large images ( $2 \mathrm{Mpixel}$ and beyond) at low compression rates (high quality).
\end{abstract}

\section{Introduction}

In this work we are looking for optimal/suboptimal solutions of sign coding in image compressors that are based in the use of the Discrete Wavelet Transform (DWT). Wavelet transforms have been widely used, since many state-of-the-art 
image codecs, including the JPEG2000 standard [17], employ DWT into their algorithms. One advantage of the wavelet transform is the provision of both frequency and spatial localization of image energy. The image energy is compacted into a small fraction of the transform coefficients and compression can be achieved by coding these coefficients. The energy of a wavelet transform coefficient is restricted to non-negative real numbers, but the coefficients themselves are not, and they are defined by both a magnitude and a sign. A transform coefficient is equally likely to be positive or negative and thus one bit should be used to encode the sign, as Shapiro stated in [16]. In recent years, several authors have begun to use context modeling for wavelet sign coding [5, 17, 19], showing that despite the equiprobability of wavelet sign values, some sign correlation can be found among wavelet coefficients, resulting in overall compression ratio improvements.

In a previous work [10] we have observed that the sign of a wavelet coefficient may be strongly correlated with the sign of some neighbor coefficients. However, this relationship is not uniform and constant for any image, or even consistent within the same image. Thus, although a careful analysis for the target image could be done in order to get the most accurate sign relationships and therefore better sign prediction and improved compression rates, these sign relationships would be only useful for this image. By increasing the number and kind of images under analysis, the relationship between the signs of the neighbor coefficients may be generalized.

Genetic algorithms (GA) were first introduced by Holland in [8] and they are nowadays well known techniques for finding nearly optimal solutions of very large problems and also, they have been used in image processing [1,3]. In a genetic algorithm, the evolution usually starts from a population of randomly generated individuals and happens in generations. In each generation, the fitness of every individual in the population is evaluated by means of a cost function that determines the optimal degree we are looking for (i.e compression rate). Multiple individuals are stochastically selected from the current population (based on their fitness), and modified (recombined and possibly randomly mutated) to form a new population. The new population is then used in the next iteration of the algorithm. Commonly, the algorithm terminates when either a maximum number of generations has been produced, or a satisfactory fitness level has been reached for the population.

In this paper we evaluate our sign coding technique proposal based on the use of genetic algorithms. We developed a genetic algorithm that will find the best sign prediction. It will work with a large set of test images in order to obtain a good sign prediction that be image independent. The evaluation will be performed over a non-embedded wavelet base encoder called LTW [13], showing the benefits of minimizing the entropy of sign information resulting from the sign prediction coding process.

The rest of the paper is organized as follows: Section 2 describes our contextbased sign coding framework and introduces a brief description about genetic algorithms and their application to sign coding prediction. In Section 3, we show 
the results of the proposed sign coding technique and its impact over the global encoder performance. Finally, in Section 4 some conclusions are drawn.

\section{Context-based sign coding approach}

Most wavelet image codecs do not consider the use of sign coding techniques since the wavelet coefficients located at the high frequency subbands form a zero-mean process, and therefore they are equally likely positive as negative.

Schwartz et al. [15] were the first authors to consider wavelet coefficient sign coding, using the sign of one neighboring pixel in their context modeling algorithm. The main idea behind this approach is to find correlations along and across edges.

The HL subbands of a multi-scale 2-D wavelet decomposition are formed from low-pass vertical filtering and high-pass horizontal filtering. The high-pass filtering detects vertical edges, thus the HL subbands mainly contain vertical edge information. Oppositely defined are the LH subbands that contain primarily horizontal edge information.

As Deever and Hemami explained in [6], given a vertical edge in an HL subband, it is reasonable to expect that neighboring coefficients along the edge have the same sign as the coefficient being coded. This is because vertical correlation often remains very high along vertical edges in images. When a low-pass filter is applied along the image columns, it results in a series of similar rows, as elements in a row tend to be very similar to elements directly above or below due to the high vertical correlation. Subsequent high-pass filtering along similar rows is expected to yield vertically correlated transform coefficients.

It is also important to consider correlation across edges, being the nature of the correlation directly affected by the structure of the high pass filter. For Daubechies' 9/7 filters, which are widely used in image compression, wavelet coefficient signs are strongly negatively correlated across edges because this filter is very similar to a second derivative of a Gaussian as derived from theory of zero crossings and edge detection [11]. So, it is expected that wavelet coefficients will change sign as the edge is crossed. Although the discrete wavelet transform involves sub sampling, the sub sampled coefficients remain strongly negatively correlated across edges. In this manner, when a wavelet coefficient is optimally predicted as a function of its across-edge neighbors (e.g. left and right neighbors in HL subbands), the optimal prediction coefficients are negative, indicating an expected sign change. This conclusion is general for any wavelet with a shape similar to a second derivative of a Gaussian.

We have applied a 6-level Dyadic Wavelet Transform decomposition of the source image and then a low quantization level to the resulting wavelet coefficients. Typically, all wavelet based image encoders apply 5 or 6 wavelet decomposition levels $[7,9,13,14]$. As a first approach and taking into account that the sign neighborhood correlation depends on the subband type (HL, LH, HH) as Deever assesses in [5], we have used three different neighbors depending on the subband type. So, for HL subband, the neighbors coefficients used to predict the sign are 
located at N (North), NN (North-North) and W (West) relative positions. Taking into account symmetry, for the LH subband, those neighbors are W (West), WW (West-West), and N (North). For the HH subband they are N (North), W (West), and NW (North-West), exploiting the correlation along and across the diagonal edges. For a particular subband, each of the three selected neighbors will have three possible sign values: positive, negative o null (zero coefficient). This lead us to a maximum of $3^{3}$ different Neighbor Sign Patterns (NSP) for each subband type.

Other encoders like JPEG2000 and the one proposed by [18] use four neighbors $(\mathrm{N}, \mathrm{S}, \mathrm{E}, \mathrm{W})$ for the context formation, but since most non-embedded encoders use a Morton order (Z-order) [12] in the coding stage, no information is available about $\mathrm{S}$ and $\mathrm{E}$ neighbors and they cannot be employed in context formation. This represents a restriction when looking for sign correlation among the neighborhood which it is shared by most of the non-embedded encoders.

Table 1: Probability distribution of neighbor sign patterns (NSPs) of $H L_{6}$ subband ( $8 x 8$ coefficients) in Lena image.

\begin{tabular}{|c|c|c|}
\hline $\mathrm{C} \mathbf{N} \mathbf{N N} \mathbf{W}$ & Occurrences & \%Probability \\
\hline$++\quad+\quad+$ & 13 & 20.31 \\
\hline+++- & 8 & 12.50 \\
\hline$-\quad-$ & 8 & 12.50 \\
\hline$+\quad+\quad+$ & 6 & 9.38 \\
\hline$-\quad+$ & 6 & 9.38 \\
\hline Others & 23 & 35.93 \\
\hline
\end{tabular}

In Table 1 we show the NSP probability distribution for $H L_{6}$ subband (from the sixth decomposition level) of Lena test image. As shown, the probability that the current coefficient (C) is positive when its $\mathrm{N}, \mathrm{NN}$ and $\mathrm{W}$ neighbors are also positive is around $20 \%$. Besides, if the $\mathrm{N}$ and $\mathrm{NN}$ neighbors have the same sign and the $\mathrm{W}$ neighbor has the opposite sign, the current coefficient $(\mathrm{C})$ has the opposite sign of its $\mathrm{W}$ neighbor with a probability of $25 \%$ as shown in rows two and three in Table 1. The visible sign neighborhood correlation suggest that the sign bits of wavelet coefficients are compressible. Using the previously mentioned neighborhood for each subband type, we have developed a genetic algorithm (GA) in order to find an accurate sign estimation.

\subsection{Genetic algorithm for wavelet sign prediction}

The goal of the desired genetic algorithm would be to find a table where for each Sign Neighborhood Pattern $\left(V_{k}\right)$ we have a sign prediction $\left(S_{i, j}\right)$ for coefficient $C_{i, j}$. There is no an univocal relationship between a neighbor sign combination, i.e not always for a same $V_{k}$ pattern, $S_{i, j}$ is always positive or negative. However, 
it is possible that for a $V_{k}$ pattern, $S_{i, j}$ is more probably to be positive or negative. But, the problem is still more complex, because a sign prediction for a neighbor sign pattern could fit well for an image and not for others. Therefore, the idea is to find suboptimal neighbor sign pattern predictions that better fit for a representative set of images.

The use of genetic algorithms to compress the sign of wavelet coefficients is twofold. First, when the number of neighbors used to analyze the sign correlation grows or when there is a great number of images to be used in the analysis, the search space is excessively wide. Second, it is not intuitive to find a way of combining the predictions obtained for several images.

In Figure 1 we show the genetic algorithm pseudo code for sign prediction. First of all we define each individual, containing a sign prediction for each $3^{3}$ NSP, then each NSP sign prediction of each individual of the universe is randomly initialized as a positive or negative sign. Then, during evolution, sequences mate and mutate to generate new individuals in the population, being selected the best ones for survival on the basis of their fitness function. The mating of sequences is performed through crossover operator, where parents are randomly selected and its gens (NSPs) are mixed. The best two individuals, the ones that exhibit best prediction performance, are selected for survival. Individuals can also undergo mutation, where a sequence prediction is randomly modified. Finally, after performing the maximum iterations, the algorithm finishes, obtaining an optimal/suboptimal sign prediction for each NSP.

Several parameters should be taken into account when training a genetic algorithm: The population size, the individuals initialization, the number of iterations performed, the mutation probability, the crossover point, the crossover method, the selection criteria of the best sequences, etc. We have performed lots of tests varying these parameters to tune the genetic algorithm [2]. The parameters used to obtain the sign prediction are: population size (100), individuals initialization (randomly), number of iterations (1000), mutation probability $(0.075 \%)$, mutation policy (inversion of a randomly chosen gen), crossover point (randomly) and crossover method (best two fitness individuals over four randomly selected parents).

The genetic algorithm takes as input data a set of test images to determine the best prediction of wavelet coefficient signs. The corresponding fitness function will score individuals whose predictions maximize sign compression rate.

After running the genetic algorithm for each subband type, we obtain an individual containing a prediction of the current coefficient sign $\left(\hat{S C_{i, j}}[k]\right)$, for each NSP $(k)$ of each subband type (see Table 2). So, what we are going to encode is the correctness of this prediction, i.e., a binary valued symbol from $\widehat{S C_{i, j}[k] \otimes S C_{i, j}}$. 


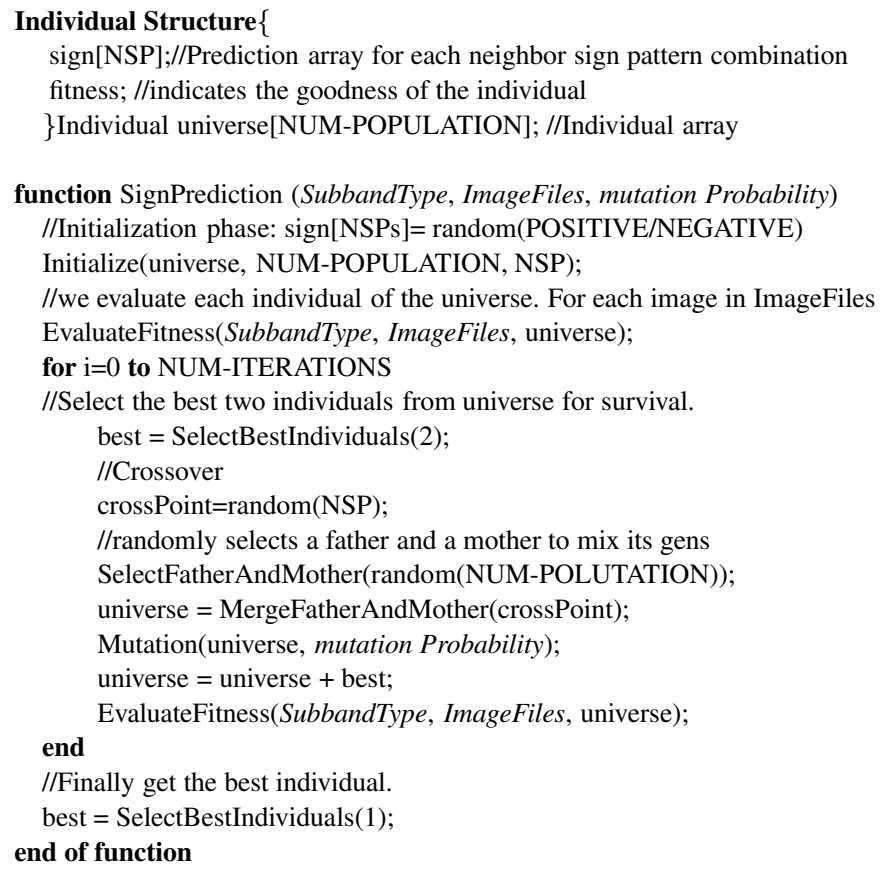

Figure 1: Genetic algorithm for sign prediction.

\section{Performance evaluation}

In this section we analyze the behavior of the proposed sign coding technique. For this purpose, we will first analyze the bit-rate savings over a set of test images of different sizes and target bit-rates. So we will evaluate the potential bit-rate reduction that whatever wavelet encoder without sign coding algorithm could obtain if our sign coding proposal is included. Before performing the experiments we have trained and tuned our proposed GA, and we have run it three times, one for each subband type over the whole Kodak image set [4] in order to get the corresponding prediction tables. Those tables were codewired in both encoder and decoder.

In order to determine the impact of the proposed sign coding technique in the encoder overall performance, we implemented our sign coding proposal in the LTW wavelet encoder [13], calling it S-LTW. After that, we performed several experimental tests comparing S-LTW encoder with LTW and SPIHT (Spiht 8.01) in terms of R/D. The test images used in the evaluation were: Lena $(512 \times 512)$, Barbara (512x512), Bike (2560x2048), GoldHill (512x512), Cafe (2560x2048),

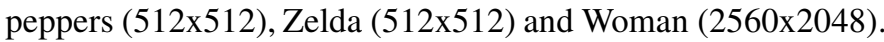

In order to determine the compression performance of the proposed sign coding scheme alone, we have selected a wavelet non-embedded encoder (LTW), and one 
Table 2: Sign prediction table for HL, LH and HH subbands. $N_{1}, N_{2}$ and $N_{3}$ are the corresponding neighbors for each subband type.

\begin{tabular}{|c|c|c|c|c|c|c|}
\hline $\mathbf{k}$ & $N_{1}$ & $\begin{array}{c}\mathbf{N S P} \\
N_{2}\end{array}$ & $N_{3}$ & 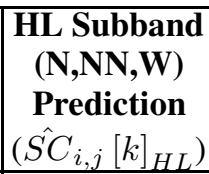 & \begin{tabular}{|c|} 
LH Subband \\
(W,WW,N) \\
Prediction \\
$\left(\hat{\left.S C_{i, j}[k]_{L H}\right)}\right.$ \\
\end{tabular} & \begin{tabular}{|c|} 
HH Subband \\
(N,W,NW) \\
Prediction \\
$\left(\hat{\left.S C_{i, j}[k]_{H H}\right)}\right)$ \\
\end{tabular} \\
\hline 0 & $*$ & $*$ & $*$ & + & + & + \\
\hline 1 & $*$ & $*$ & + & - & - & + \\
\hline 2 & $*$ & $*$ & - & + & + & - \\
\hline 3 & $*$ & + & $*$ & + & + & - \\
\hline 4 & $*$ & + & + & - & - & - \\
\hline 5 & $*$ & + & - & + & + & - \\
\hline 6 & $*$ & - & $*$ & - & - & + \\
\hline 7 & $*$ & - & + & - & - & + \\
\hline 8 & $*$ & - & - & + & + & + \\
\hline 9 & + & $*$ & $*$ & + & - & - \\
\hline 10 & + & $*$ & + & - & - & - \\
\hline 11 & + & $*$ & - & + & + & - \\
\hline 12 & + & + & $*$ & + & + & - \\
\hline 13 & + & + & + & - & - & - \\
\hline 14 & + & + & - & + & + & - \\
\hline 15 & + & - & $*$ & - & - & + \\
\hline 16 & + & - & + & - & - & - \\
\hline 17 & + & - & - & + & + & + \\
\hline 18 & - & $*$ & $*$ & - & + & + \\
\hline 19 & - & $*$ & + & - & - & + \\
\hline 20 & - & $*$ & - & + & + & + \\
\hline 21 & - & + & $*$ & + & + & + \\
\hline 22 & - & + & + & - & - & - \\
\hline 23 & - & + & - & + & + & + \\
\hline 24 & - & - & $*$ & - & - & + \\
\hline 25 & - & - & + & - & - & + \\
\hline 26 & - & - & - & + & + & + \\
\hline
\end{tabular}

embedded encoder like SPIHT. As no sign coding technique is used, the sign information is raw encoded, so it would require so many bits as the number of significant coefficients (one bit per significant coefficient). The sign coding results were obtained for different image target bit-rates, counting the total number of significant coefficients to be encoded just after quantization. For example, the LTW encoder at 1 bpp has to encode 45,740 significant coefficients requiring 45,740 bits for coding sign information. If we include the proposed sign encoding technique the encoded sign information will be reduced to 37,804 bits, representing a $17.35 \%$ of bit-rate savings. To show the savings on the other selected wavelet encoder (SPIHT), we proceed in a similar way. First we obtain the total number of 
Table 3: Sign compression performance at different bit-rates for S-LTW and SPIHT.

\begin{tabular}{|c||cc|cc||c|}
\hline \multicolumn{1}{|c||}{$\begin{array}{c}\text { Bit-rate } \\
\text { (bpp) }\end{array}$} & $\begin{array}{c}\text { S-LTW } \\
\text { \#Significant }\end{array}$ & $\begin{array}{c}\text { S-SPIHT } \\
\text { \#-Sits }\end{array}$ & \#Significant & \#Bits & \\
Coefficients & Saved & Coefficients & Saved & \\
\hline \multicolumn{5}{|c|}{ Barbara (512x512) } \\
\hline 1 & 45740 & 7936 & 54657 & 9482 & $\mathbf{1 7 . 3 5}$ \\
0.5 & 22331 & 3648 & 27535 & 4499 & 16.34 \\
0.25 & 10484 & 1520 & 13460 & 1951 & 14.50 \\
0.125 & 4343 & 304 & 6016 & 421 & 7.00 \\
\hline \multicolumn{6}{|c|}{ Bike (2048x2560) } \\
\hline 1 & 855266 & 115200 & 1371280 & 184711 & 13.47 \\
0.5 & 412212 & 64424 & 798202 & 124758 & 15.63 \\
0.25 & 198943 & 30472 & 366927 & 56213 & 15.32 \\
0.125 & 91767 & 11992 & 162990 & 21302 & 13.07 \\
\hline \multicolumn{7}{|c|}{ Lena (512x512) } \\
\hline 1 & 51113 & 4032 & 51883 & 4093 & 7.89 \\
0.5 & 20886 & 2328 & 25451 & 2837 & 11.15 \\
0.25 & 10038 & 880 & 12651 & 1109 & 8.77 \\
0.125 & 4724 & 256 & 6204 & 336 & 5.42 \\
\hline
\end{tabular}

significant coefficients after quantization. Then we perform a lineal estimation of bits saved, assuming that there is the same relative gain than in LTW, since both encoders use the same DWT filter.

In Table 3 we show the relative compression gains with respect to the original encoder due only to the sign coding capability for several test images. As we can see, the maximum sign compression gain is $17.35 \%$ for Barbara image at $1 \mathrm{bpp}$. As expected, the compression gain is higher at low compression rates because the sign prediction model has been performed using all wavelet coefficients sign information. As the compression rate increases, the number of non-significant coefficients increase, loosing prediction performance since the NSPs with some non-significant neighbour would be more and more dominant what it is just the opposite situation when computing the sign prediction table. This effect results in lowering the compression gains up to a $5.42 \%$ for Lena image at $0.125 \mathrm{bpp}$.

\section{Conclusions}

We have presented a study about sign coding for non-embedded image encoders. We propose a simplified context model formation that it is oriented to maximize the successful prediction of the sign for every significant wavelet coefficient. In order to obtain a good sign prediction, we employ a genetic algorithm specially trained and tuned with a representative image set. The prediction result is encoded with an adaptive arithmetic encoder to compact the sign information as much as 
possible. We have implemented it over the LTW encoder in order to evaluate the sign context model behavior. The new proposed sign coding technique is able to reduce the sign information with a compression gain up to $17.35 \%$ which lead in a R/D performance increase up to $0.37 \mathrm{~dB}$ (as experimental results show), being greater the improvement at low and medium compression rates. Also, we have shown that other encoders like SPIHT will obtain similar improvements when including the sign prediction model, being directly applicable to whatever nonembedded wavelet encoder.

\section{Acknowledgements}

Thanks to Spanish Ministry of Education and Science under grants TIN201127543-C03-03 and TEC2010-11776-E for funding.

\section{References}

[1] Sarawat Anam, Md. Shohidul Islam, M.A. Kashem, M.N. Islam, M.R. Islam, and M.S. Islam. Face recognition using genetic algorithm and back propagation neural network. In International MultiConference of Engineers and Computer Scientists, Hong Kong, 2009.

[2] A. Martí Campoy, F. Rodríguez-Ballester, O. López, and M.P. Malumbres. An embedded image coding system baed on tarp filter with classification. In Proc. IADIS International Conference on Applied Computing, Madrid, Spain, October 2012.

[3] S. Chabrier, C. Rosenberger, B. Emile, , and H. Laurent. Optimization-based image segmentation by genetic algorithms. EURASIP Journal on Image and Video Processing, 2008:1-10, 2008.

[4] CIPR. http://www.cipr.rpi.edu/resource/stills/kodak.html. Center for image processing research.

[5] Aaron Deever and Sheila S. Hemami. What's your sign?: Efficient sign coding for embedded wavelet image coding. In Proc. IEEE Data Compression Conf., Snowbird, UT, pages 273-282, 2000.

[6] Aaron Deever and Sheila S. Hemami. Efficient sign coding and estimation of zero-quantized coefficients in embedded wavelet image codecs. IEEE Transactions on Image Processing, 12(4):420-431, April 2003.

[7] Jiangling Guo, Sunanda Mitra, Brian Nutter, and Tanja Karp. A fast and low complexity image codec based on backward coding of wavelet trees. Data Compression Conference, 2006.

[8] J.H. Holland. Adaption in Natural and Artificial Systems. University of Michigan Press, 1975.

[9] ISO/IEC 15444-1. JPEG2000 image coding system, 2000. 
[10] O. Lopez, M. Martinez, P. Pinol, M.P. Malumbres, and J. Oliver. E-LTW: An enhanced ltw encoder with sign coding and precise rate control. In Image Processing (ICIP), 2009 16th IEEE International Conference on, pages 2821 -2824 , Nov. 2009.

[11] Stephane Mallat and Sifen Zhong. Characterization of signals from multiscale edges. IEEE Transactions on Pattern Analysis and Machine Intelligence, 14(7):710-732, July 1992.

[12] G. M. Morton. A computer oriented geodetic data base and a new technique in file sequencing. Technical report, IBM Ltd., 1966.

[13] J. Oliver and M. P. Malumbres. Low-complexity multiresolution image compression using wavelet lower trees. IEEE Transactions on Circuits and Systems for Video Technology, 16(11):1437-1444, 2006.

[14] A. Said and A. Pearlman. A new, fast and efficient image codec based on set partitioning in hierarchical trees. IEEE Transactions on Circuits, Systems and Video Technology, 6(3):243-250, 1996.

[15] Edward L. Schwartz, Ahmad Zandi, and Martin Boliek. CREW: Compression with reversible embedded wavelets. In In Proc SPIE, pages 212-221, 1995.

[16] J.M. Shapiro. A fast technique for identifying zerotrees in the EZW algorithm. Proc. IEEE Int. Conf. Acoust., Speech, Signal Processing, 3:1455$1458,1996$.

[17] D. Taubman. High performance scalable image compression with EBCOT. IEEE Transactions on Image Processing, 9(7):1158-1170, July 2000.

[18] C. Tian and Sheila S. Hemami. An embedded image coding system baed on tarp filter with classification. In Proc. IEEE International Conference on Acoustics, Speech, and Signal Processing (ICASSP), Montreal, Canada, May 2004.

[19] X. Wu. High-order context modeling and embedded conditional entropy coding of wavelet coefficients for image compression. In Proc. of 31st Asilomar Conf. on Signals, Systems, and Computers, pages 1378-1382, 1997. 